\title{
A randomised-controlled trial of the effects of very low-carbohydrate and high-carbohydrate diets on cognitive performance in patients with type 2 diabetes
}

\author{
Jeannie Tay ${ }^{1,2,3}$, Ian T. Zajac ${ }^{1}$, Campbell H. Thompson ${ }^{2}$, Natalie D. Luscombe-Marsh ${ }^{1}$, Vanessa Danthiir ${ }^{1}$, \\ Manny Noakes ${ }^{1}$, Jonathan D. Buckley ${ }^{4}$, Gary A. Wittert ${ }^{2}$ and Grant D. Brinkworth ${ }^{1}$ * \\ ${ }^{1}$ Commonwealth Scientific and Industrial Research Organisation (CSIRO) - Health and Biosecurity, Adelaide, SA 500O, Australia \\ ${ }^{2}$ Discipline of Medicine, University of Adelaide, Adelaide, SA 5000, Australia \\ ${ }^{3}$ Agency for Science, Technology and Research (A-STAR), Singapore 138632 \\ ${ }^{4}$ Alliance for Research in Exercise, Nutrition and Activity (ARENA), Sansom Institute for Health Research, \\ University of South Australia, Adelaide, SA 5001, Australia
}

(Submitted 24 May 2016 - Final revision received 22 September 2016 - Accepted 19 October 2016)

\section{Abstract}

This study compared the longer-term effects of a very low-carbohydrate, high-fat diet with a high-carbohydrate, low-fat diet on cognitive performance in individuals with type 2 diabetes (T2D). In total, 115 obese adults with T2D (sixty-six males, BMI: 34.6 (sD 4.3) kg/m², age: 58 (sD 7) years, HbA1c: $7 \cdot 3$ (sD 1.1)\%, diabetes duration: 8 (SD 6) years) were randomised to consume either an energy-restricted, very lowcarbohydrate, low-saturated-fat (LC) diet or an energy-matched high unrefined carbohydrate, low-fat (HC) diet with supervised aerobic/ resistance exercise ( $60 \mathrm{~min}, 3 \mathrm{~d} /$ week) for 52 weeks. Body weight, HbA1c and cognitive performance assessing perceptual speed, reasoning speed, reasoning ability, working memory, verbal fluency, processing speed, short-term memory, inhibition and memory scanning speed were assessed before and after intervention. No differences in the changes in cognitive test performance scores between the diet groups were observed for any of the cognitive function outcomes assessed $(P \geq 0.24$ time $\times$ diet). Percentage reduction in body weight correlated with improvements with perceptual speed performance. In obese adults with T2D, both LC and HC weight-loss diets combined with exercise training had similar effects on cognitive performance. This suggests that an LC diet integrated within a lifestyle modification programme can be used as a strategy for weight and diabetes management without the concern of negatively affecting cognitive function.

\section{Key words: Diabetes: Macronutrient composition: Cognitive performance: Weight loss: Glycaemic control}

The rising prevalence of obesity and type 2 diabetes (T2D) has fuelled interest in very low-carbohydrate diets (LC: 20-70 g carbohydrates/d). This is supported by current dietary guidelines advocating personalised management ${ }^{(1)}$, and increasing evidence demonstrating the long-term efficacy of LC diets for improving glycaemic control and cardiometabolic outcomes compared with traditional high-carbohydrate (HC) diets ${ }^{(2-4)}$.

T2D increases the risk for cognitive impairment and dementia ${ }^{(5,6)}$. Psychomotor efficiency, learning, executive functioning, memory and speed of information processing are often most affected ${ }^{(5,7)}$. This underscores the importance of considering the effect of diabetes management strategies on cognitive function. A concern with LC diets is that carbohydrate restriction below the recommended daily allowance of $130 \mathrm{~g} / \mathrm{d}$, corresponding to the average minimum amount utilised by the brain $^{(8)}$, could impair cognitive function by lowering dietary glucose availability. Consequently, there are concerns that LC diets may adversely affect cognitive function ${ }^{(9)}$.

A limited number of studies have examined the effects of LC diets on cognitive function beyond a few weeks. To date, only two longer-term (24-52 week) studies have been conducted, and these reported no differences in cognitive performance between participants consuming either an LC or HC diet ${ }^{(10,11)}$. However, these studies only examined a limited range of cognitive domains in individuals without T2D. It is therefore critical to examine the longer-term effects of LC diets on cognitive performance on a more comprehensive range of cognitive domains in individuals with T2D. This study aimed to compare the effects of a hypoenergetic LC diet with an energy-matched HC diet administered as part of a lifestyle modification programme on cognitive performance in obese individuals with T2D after 52 weeks. It was hypothesised

Abbreviations: DSST, digit symbol substitution; HC, high carbohydrate, low-fat diet; LC, very low-carbohydrate, high-unsaturated/low-saturated-fat diet; T2D, type 2 diabetes.

* Corresponding author: Associate Professor G. D. Brinkworth, fax +61 88303 8899, email grant.brinkworth@csiro.au 
that, compared with a HC diet, an LC diet would reduce cognitive performance.

\section{Methods \\ Study design and participants}

The study was conducted at the CSIRO Clinical Research Unit between May 2012 and September 2013. The participants and study design have been described elsewhere ${ }^{(4)}$. In brief, 115 overweight/obese adults (forty-nine females/sixty-six males) with T2D (mean age 58 (sD 7) years; HbA1c $7 \cdot 3$ (sD 1.1)\% (56 (sD 12) $\mathrm{mmol} / \mathrm{mol}$ ); BMI: 34.6 (sD $4 \cdot 3) \mathrm{kg} / \mathrm{m}^{2}$; diabetes duration 8 (SD 6 ) years; highest level of education (15\% high school graduate, $35 \%$ diploma/vocational training, $50 \%$ university/higher degree)) were recruited by public advertisement. In total, twelve participants were taking insulin medication, eighty-seven were taking metformin, thirty-six were taking sulfonylureas, six were taking thiazolidinedione, two were taking glucagon-like peptide- 1 agonists and three were taking dipeptidyl peptidase- 4 medication. The exclusion criteria included type 1 diabetes; abnormal renal or liver function; any significant endocrinopathy (other than stable treated thyroid disease); history of malignancy or respiratory, gastrointestinal, cerebrovascular, peripheral or CVD; pregnancy or lactation; severe depression and current depression (Beck Depression Inventory Score $\geq 29$ ); history of/or current eating disorder; or smoking. The study was conducted according to the guidelines laid down in the Declaration of Helsinki and the protocol and all procedures were approved by the Human Research Ethics Committees of the Commonwealth Scientific and Industrial Research Organisation (CSIRO), the University of Adelaide and the University of South Australia. Before study commencement, participants provided written informed consent.

The study used a 52-week parallel design and participants were block-matched for sex, BMI, age, HbA1c and diabetes medication before they were randomly allocated (by computer generation) to consume either a hypoenergetic $(2092-4184 \mathrm{~kJ} / \mathrm{d}$ deficit (500-1000 kcal/d deficit)) LC, high-unsaturated/ low-saturated-fat diet ( $n$ 57; $14 \%$ energy as carbohydrate $(\mathrm{CHO}<50 \mathrm{~g} / \mathrm{d}), 28 \%$ protein $(\mathrm{PRO}), 58 \%$ fat $(<10 \% \mathrm{SFA}))$ or an energy-matched HC diet ( $n 58 ; 53 \%$ CHO, $17 \%$ PRO, $30 \%$ fat $(<10 \% \mathrm{SFA})$ ) that reflected conventional dietary guidelines ${ }^{(12)}$, in a 1:1 ratio. Research associates not involved in the data collection or delivery of the intervention performed the randomisation procedures that included sequence generation and allocation concealment. To achieve the targeted macronutrient profile, specific foods and quantities were listed in a food record that was completed daily by participants. Diet plans were personalised for energy requirements and participants received dietetic counselling biweekly for the first 12 weeks, and monthly for the remainder of the study. To facilitate compliance, participants were provided with key foods (approximately 30\% total energy) that reflected the assigned diet profiles for the initial 12 weeks, and for the remainder of study were provided with key foods or a \$50AUD voucher on alternating months. All participants also undertook the same supervised moderate-intensity aerobic/resistance exercise sessions (60 min, 3d/week), consistent with diabetes management guidelines ${ }^{(13)}$. These group-based exercise classes were conducted in local community centres, and participants from both diet groups attended classes together.

Detailed description of the diets, exercise programme and the effects of the interventions on weight, glycaemic control and cardiometabolic outcomes have been previously reported ${ }^{(4)}$. Both groups achieved high dietary compliance and had similar increases in physical activity levels ${ }^{(4)}$. Exercise-session attendance was similar in both groups (LC diet: 81.2 (SD 18.0)\%; HC diet: 77.5 (sD 21.6) \%; $P=0.47$ ). Compared with HC participants, LC participants reported a lower mean carbohydrate intake (LC 54-74 g/d; HC 202-218 g/d).

\section{Outcome measures}

Outcome measures were assessed at baseline (week 0) and at weeks 24 and 52. Body height and weight (assessed monthly) was assessed using a stadiometer (SECA) and electronic scales (Mercury AMZ1), respectively. HbA1c was assessed at a certified pathology laboratory (SA Pathology). The test battery used to assess cognitive performance (Table 1 ) represented a subset of a previous test battery administered in a clinical study examining the effects of diet on cognitive function ${ }^{(16)}$. The present battery comprised several tasks measuring a broad variety of cognitive domains. Tests were selected because they assess cognitive domains most consistently affected by diabetes and have been shown to be sensitive to nutritional interventions $^{(6,17-19)}$. Specifications of each test have been fully described elsewhere ${ }^{(16)}$.

Cognitive assessments were conducted at CSIRO's cognitive laboratory after overnight fasting and water consumed as required. No hypoglycaemic incidents were reported. Tests were administered in a consistent order across test sessions and scored by trained research personnel blinded to participants' treatment assignment. Each session included computer (Inquisit version 2; Millisecond Software) and paper tasks lasting approximately $1 \mathrm{~h}$. The tasks were administered in the same order as listed in Table 1, with speed and accuracy-based tasks interspersed to reduce the likelihood of fatigue.

Each task was preceded by practice items and queries were addressed before test commencement. No colour blindness was reported. Parallel versions were used for those tasks (word memory, operation span and word endings tests) in which the stimuli might feasibly be remembered between sessions. All other tasks are arguably more process-driven and preclude the 'learning' of stimuli, and thus the same version of the test was administered at each assessment ${ }^{(16)}$. The average test-retest reliabilities were high and acceptable for most tasks, as shown in Table 1, although reliabilities were lower than anticipated for colour Stroop and word memory tasks.

Quality control included regular audit of test sessions, independent dual-scoring and regular review of session notes. Depression, a potential confounder and frequent comorbidity in T2D, was assessed by the Beck Depression Inventory-II (BDI-II) ${ }^{(20)}$ to control for possible effects of mood disturbances or affective disorders. Participants' BDI-II scores were in the subclinical range (mean LC $6 \cdot 3$ (sD 6.2); HC 5.2 (sD 4.7), $P=0.29$, Table 2). 
Table 1. Cognitive test battery

\begin{tabular}{|c|c|c|c|c|}
\hline Tasks & Cognitive domain & Task description & Outcome measure & $\begin{array}{l}\text { Mean test-retest } \\
\text { reliability } \dagger\end{array}$ \\
\hline \multicolumn{5}{|l|}{ Computer-based tasks } \\
\hline Odd-man-out letter task & Reasoning speed & $\begin{array}{l}\text { Participants decide as quickly as possible the whereabouts } \\
\text { of the 'odd-man-out' in a set of letters }\end{array}$ & Average work rate (1000/mean response latency) & 0.74 \\
\hline Word memory & Short-term memory & $\begin{array}{l}\text { Fifteen words (nouns) are presented one at a time on the } \\
\text { computer screen. After presentation of all words, participants } \\
\text { write down as many as they can recall, in any order }\end{array}$ & $\begin{array}{c}\text { Number of words correctly recalled from } \\
\text { presented list (maximum score of } 15 \text { ) }\end{array}$ & 0.53 \\
\hline Operation span & Working memory & $\begin{array}{l}\text { Participants remember words while simultaneously completing } \\
\text { arithmetic problems. There are different block lengths (from } \\
\text { two words through to six words) }\end{array}$ & $\begin{array}{l}\text { The number of words recalled correctly in the } \\
\text { correct position within corresponding blocks }\end{array}$ & 0.78 \\
\hline Two-choice reaction time & Processing speed & $\begin{array}{l}\text { Participants respond as quickly as possible to on-screen stimuli } \\
\text { (the numbers ' } 1 \text { ' or '2') by pressing the left or right arrows on } \\
\text { the numeric keypad of the keyboard }\end{array}$ & Average work rate (1000/mean response latency) & 0.72 \\
\hline Number memory scanning & $\begin{array}{l}\text { Speed of memory } \\
\text { scanning }\end{array}$ & $\begin{array}{l}\text { A string of numbers is presented one at a time. After the } \\
\text { presentation string, a target number is shown on screen. } \\
\text { Participants indicate as quickly as possible whether } \\
\text { the number was in the sequence presented } \\
\text { immediately before }\end{array}$ & Average work rate (1000/mean response latency) & 0.73 \\
\hline Colour Stroop & Inhibition & $\begin{array}{l}\text { Participants press the right shift key on the keyboard if the } \\
\text { word is coloured blue, and the left shift key if it is coloured } \\
\text { yellow. Words are presented in congruent (word matches the } \\
\text { printed colour), incongruent (word does not match the printed } \\
\text { colour) or neutral format (neutral words presented) }\end{array}$ & $\begin{array}{l}\text { Difference between work rates }(1000 / \text { mean } \\
\text { response latency): incongruent trial work rate } \\
\text { minus mean work rate across congruent and } \\
\text { neutral trials ( } 30 \text { each) }\end{array}$ & 0.45 \\
\hline \multicolumn{5}{|l|}{ Paper and pencil-based tasks } \\
\hline $\begin{array}{l}\text { Digit-symbol substitution } \\
\text { test (DSST) }\end{array}$ & Perceptual speed & $\begin{array}{l}\text { A coding key is presented at the top of a page showing the } \\
\text { numbers } 1-9 \text {, each paired with a unique symbol. Participants } \\
\text { copy as many symbols as possible corresponding to a random } \\
\text { array of numbers }{ }^{(14)}\end{array}$ & Number of items completed correctly in $90 \mathrm{~s}$ & 0.83 \\
\hline Word endings & Verbal fluency & $\begin{array}{l}\text { Participants are given } 2 \text { min to freely recall as many words as } \\
\text { possible, which end with a prescribed set of letters } \\
\text { (e.g. '-ate') })^{(15)}\end{array}$ & $\begin{array}{l}\text { Number of freely recalled words in } 2 \text { min with a } \\
\text { specified ending }\end{array}$ & 0.63 \\
\hline Letter sets & Reasoning ability & $\begin{array}{l}\text { Participants analyse five sets of four letters in order to determine } \\
\text { the common pattern amongst the sets, and to decide which set } \\
\text { does not match the recurring pattern }{ }^{(15)}\end{array}$ & Number of items out of fifteen answered correctly & 0.78 \\
\hline
\end{tabular}

* Tasks represent a sub set from Danthiir et al.(16)

† Test-retest reliabilities represent the average reliability across sessions and is calculated as $M r=\operatorname{Mean}\left(r_{1,2}, r_{1,3}, r_{2,3}\right)$. 
Eight LC participants reported taking antidepressant medications (ADM) and two HC participants began taking $\mathrm{ADM}$ during the study. None of the participants were on dementia medications or taking any cognitive enhancing drugs or nootropics.

\section{Statistical analysis}

The initial sample size calculation for this study was based on the primary study outcome of HbA1c, as previously reported ${ }^{(4)}$. However, a priori power analyses were also conducted to determine the power to detect clinically relevant differences between groups for cognitive function measures using G*Power software $^{(21)}$. For a univariate repeated measures model, we determined that a minimum combined sample size of $n 82$ was necessary to detect a small $(f=0 \cdot 15$, equivalent to Cohen's $d=$ approximately 0.30 ) time $\times$ diet interaction effect with $95 \%$ power, assuming $\alpha=0.05$ for the cognitive function tests and correlation among measures of $r 0 \cdot 66$. The minimum number required to retain $80 \%$ power to detect the same time $\times$ diet small interaction effect $(f=0.15, \alpha=0.05, r \quad 0.66)$ is $n 64$. Therefore, the sample size that completed the present study ( $n$ 78) was suitable to assess the cognitive outcome measures.

Baseline demographic and clinical characteristics between the groups were compared using independent $t$ tests for continuous variables and $\chi^{2}$ tests for categorical variables. Mean response latencies for speed tasks were converted to work rates (1000/mean latency) in order to normalise the distributions. Work rates represent the average number of items completed per second of measured response time, and higher scores indicate better performance. The effect of the diets on changes in cognitive function over time was analysed by random-coefficient analysis, restricted maximum likelihood and mixed effects models using an unstructured covariance that assumed data to be missing at random. Participants with a cognitive assessment at baseline and at least 24 or 52 weeks of follow-up were included in the final analyses ( $n 114$ for word memory, two-choice reaction time and odd-man-out letter (OMO); $n 113$ for digit symbol substitution (DSST), word endings, number memory scanning (MScan) and colour Stroop; $n 112$ for letter sets and operation span tasks). Four (LC: three, HC: one) participants were excluded from various cognitive function analyses because of non-compliance to the test protocol, accuracy score $<50 \%$, language difficulty and time constraints precluding completion of tests. Mean test-retest reliability representing the average reliability across sessions was calculated as $\mathrm{M} r=\operatorname{Mean}\left(r_{1,2}, r_{1,3}, r_{2,3}\right)$. The following fixed effects were included in the model: main effect for each time point, diet group assignment and diet group by time point interaction. Estimated marginal means (95\% CI) and change from week 0 to 52 are reported. Analyses with adjustment for sex, age, depression and education status were conducted for sensitivity analyses. The magnitudes of effect size (d) for time effects were calculated as the mean difference in performance from week 0 to week 52 divided by the pooled SD of the difference $^{(22)}$. Simple linear regression analysis was used to determine relationships between the change in anthropometric markers (weight and BMI) and changes in cognitive function. For cognitive variables, absolute change scores were calculated and used as the difference score (week 52-week 0), and weight loss was expressed as percentage of total weight loss $(($ difference score/weight at week 0$) \times 100)$. Where significant univariate effects were found, multivariate models were used to adjust for the impact of sex and age. SPSS 20.0 for Windows (SPSS Inc.) was used to perform the analyses and statistical tests were two-tailed with $P<0.05$ considered statistically significant.

\section{Results}

Baseline characteristics were not significantly different between diet groups $(P>0.05$, Table 2$)$. As previously reported ${ }^{(4)}$, the two groups had similar rates of study completion (LC $71 \%$, HC $65 \%, P=0.51$ ) (Fig. 1), reductions in weight (mean LC -9.8 ; $95 \% \mathrm{CI}-11 \cdot 7,-7 \cdot 9 ; \mathrm{HC}-10 \cdot 1 ; 95 \% \mathrm{CI}-12 \cdot 0,-8 \cdot 2 \mathrm{~kg}, P=0 \cdot 18)$ and HbA1c (LC $-1 \cdot 0 ; 95 \% \mathrm{CI}-1 \cdot 2,-0 \cdot 7$; HC $-1 \cdot 0$; $95 \% \mathrm{CI}-1 \cdot 3$, $-0.8 \%, P=0.65)$.

Cognitive outcomes based on estimated marginal means from primary mixed-models analyses are shown in Table 3. No statistically significant differences in cognitive test performance scores between the diet groups over time for any of the cognitive outcomes assessed were observed ( $P \geq 0.24$ time $\times$ diet). Some main effects of time were observed. Specifically, word endings (Cohen's $d=1 \cdot 01, P<0 \cdot 001$ ) and word memory scores (Cohen's $d=0.31, P=0.005)$ decreased over time, whereas performance on DSST (Cohen's $d=-0 \cdot 28, P=0 \cdot 03$ ), MScan (Cohen's $d=-0.34, P<0 \cdot 001$ ) and OMO performance (Cohen's $d=-0.49, \quad P<0.001)$ improved over time. Fully adjusted models controlling for covariates associated with cognitive function (age, sex, education level and depression) did not alter the results of the responses between the diet groups. Removal of the participants taking ADM did not change the outcomes.

Linear regressions showed a higher percentage change in body weight (corresponding to a greater reduction) significantly correlated to the change (increase) in DSST performance $(\beta=0.25, \quad P=0.03)$. This effect remained significant after controlling for age and sex $(\beta=0.23, P=0.03)$.

\section{Discussion}

This study showed after participation in a 52-week lifestyle intervention that both an LC diet and a HC diet had comparable effects on cognitive performance in overweight and obese individuals with T2D, as assessed by a comprehensive neuropsychological test battery. This is consistent with previous longer-term studies (24-52 weeks) conducted in populations without T2D that showed no difference in cognitive effects following consumption of LC and HC weight-loss diets on tasks that assessed working memory and speed of processing ${ }^{(10,11)}$, reaction time and attention, problem-solving and short-term memory $^{(11)}$. Results from smaller, shorter-term studies (3-8 weeks), however, do suggest some differences between $\mathrm{HC}$ and LC diets for speed of processing, attention and memory performance ${ }^{(23,24)}$. While these studies suggest that differences in cognitive performance may occur between HC and LC diets over the short term, these effects are unlikely to be sustained over a longer period, as demonstrated by the present results. 
Table 2. Baseline characteristics of participants by diet assignment ${ }^{\star}$ (Mean values and standard deviations; numbers and percentages)

\begin{tabular}{|c|c|c|c|c|c|}
\hline & \multicolumn{2}{|c|}{ LC $\operatorname{diet}(n 58)$} & \multicolumn{3}{|c|}{ HC diet ( $n 57)$} \\
\hline & Mean & SD & Mean & & SD \\
\hline \multicolumn{6}{|l|}{ Demographics } \\
\hline Age (years) & 58 & 7 & 58 & & 7 \\
\hline \multicolumn{6}{|l|}{ Sex } \\
\hline \multicolumn{6}{|l|}{ Females } \\
\hline$n$ & \multicolumn{2}{|c|}{21} & \multicolumn{3}{|c|}{28} \\
\hline$\%$ & \multicolumn{2}{|c|}{36} & \multicolumn{3}{|c|}{49} \\
\hline \multicolumn{6}{|l|}{ Males } \\
\hline$n$ & \multicolumn{2}{|c|}{37} & \multicolumn{3}{|c|}{29} \\
\hline$\%$ & \multicolumn{2}{|c|}{64} & \multicolumn{3}{|c|}{51} \\
\hline Duration of diabetes (years) & 7 & 5 & 9 & & 7 \\
\hline \multicolumn{6}{|l|}{ Education† } \\
\hline \multicolumn{6}{|l|}{ High school graduate } \\
\hline$n$ & \multicolumn{2}{|c|}{5} & \multicolumn{3}{|c|}{10} \\
\hline$\%$ & \multicolumn{2}{|c|}{9} & \multicolumn{3}{|c|}{18} \\
\hline \multicolumn{6}{|l|}{ Diploma or vocational training } \\
\hline$n$ & \multirow{2}{*}{\multicolumn{2}{|c|}{14}} & \multicolumn{3}{|c|}{20} \\
\hline$\%$ & 24 & & \multicolumn{3}{|c|}{35} \\
\hline \multicolumn{6}{|l|}{ University or higher degree } \\
\hline$n$ & \multicolumn{2}{|c|}{27} & \multicolumn{3}{|c|}{22} \\
\hline$\%$ & \multicolumn{2}{|c|}{47} & \multicolumn{3}{|c|}{39} \\
\hline \multicolumn{6}{|l|}{ Body weight and $\mathrm{BMI}$} \\
\hline Body weight (kg) & $101 \cdot 7$ & 14.4 & $101 \cdot 6$ & & $15 \cdot 8$ \\
\hline $\mathrm{BMI}\left(\mathrm{kg} / \mathrm{m}^{2}\right)$ & $34 \cdot 2$ & 4.5 & $35 \cdot 1$ & & $4 \cdot 1$ \\
\hline \multicolumn{6}{|l|}{ Glycaemic control } \\
\hline $\mathrm{HbA1c}(\%)$ & $7 \cdot 3$ & 1.1 & 7.4 & & 1.1 \\
\hline Depression & & & & & \\
\hline BDI-II score ${ }^{(20)} \ddagger$ & $6 \cdot 3$ & $6 \cdot 2$ & $5 \cdot 2$ & & 4.7 \\
\hline
\end{tabular}

Despite the lack of any differential effect of diet composition on cognitive outcomes, significant improvements in performance on the DSST, MScan and OMO tasks were observed over 52 weeks in both groups. Because of the absence of a nonintervention control group, it is likely that the improvements over time reflect practice effects ${ }^{(25,26)}$, which are relatively common for cognitive testing ${ }^{(27)}$, and are probably not due to the effects of the lifestyle interventions tested. However, it cannot be dismissed that the improvements observed may be attributed to increases in physical activity following participation in the planned exercise sessions, combined with the sustained weight loss and metabolic improvements resulting from the intervention. Previous studies have shown that exercise and particularly resistance training improves cognitive outcomes including information-processing speed, attention, memory formation and other forms of executive function ${ }^{(28-30)}$. In the present study, only improvements in speed-related cognitive domains were observed. These differences may be attributed to differences in study populations (healthy elderly with intact cognition $v$. those with mild cognitive impairment), and the intensity and frequency of the exercise prescriptions. However, as the study design was intended to compare differences in cognitive function between diet groups and the exercise intervention involved combined aerobic and resistance training that was implemented in both groups, the independent effect of resistance training on cognition cannot be elucidated.

Further, a significant relationship between the change in percentage body weight and DSST was also observed. Witte et al. ${ }^{(31)}$ showed that cognitive performance improved in overweight individuals following 3 months of energy intake restriction, and weight loss was associated with improved metabolic control compared with no improvement in a nondieting control group. Given that any practice effects would have been similar in both groups, the experimental design of the present study would be appropriate to test the hypothesis of comparing the effects of consuming either an LC or HC diet on cognitive function. Further studies that specifically examine the effect of weight loss and lifestyle modification should be conducted to determine the potential clinical benefits of this intervention strategy in populations with $\mathrm{T} 2 \mathrm{D}$ who are at an increased risk of cognitive impairment.

An interesting finding in the present study was the significant decline in performance on the word endings test. Parallel forms of this test were used and they were designed to be of equivalent difficulty. However, these scores may have decreased at follow-up assessments because of increased test complexity. Evidence of this is apparent in the magnitude of this effect; performance decreased by $1 \mathrm{sD}$, which is a very large effect. While other prospective studies have shown a decline in 


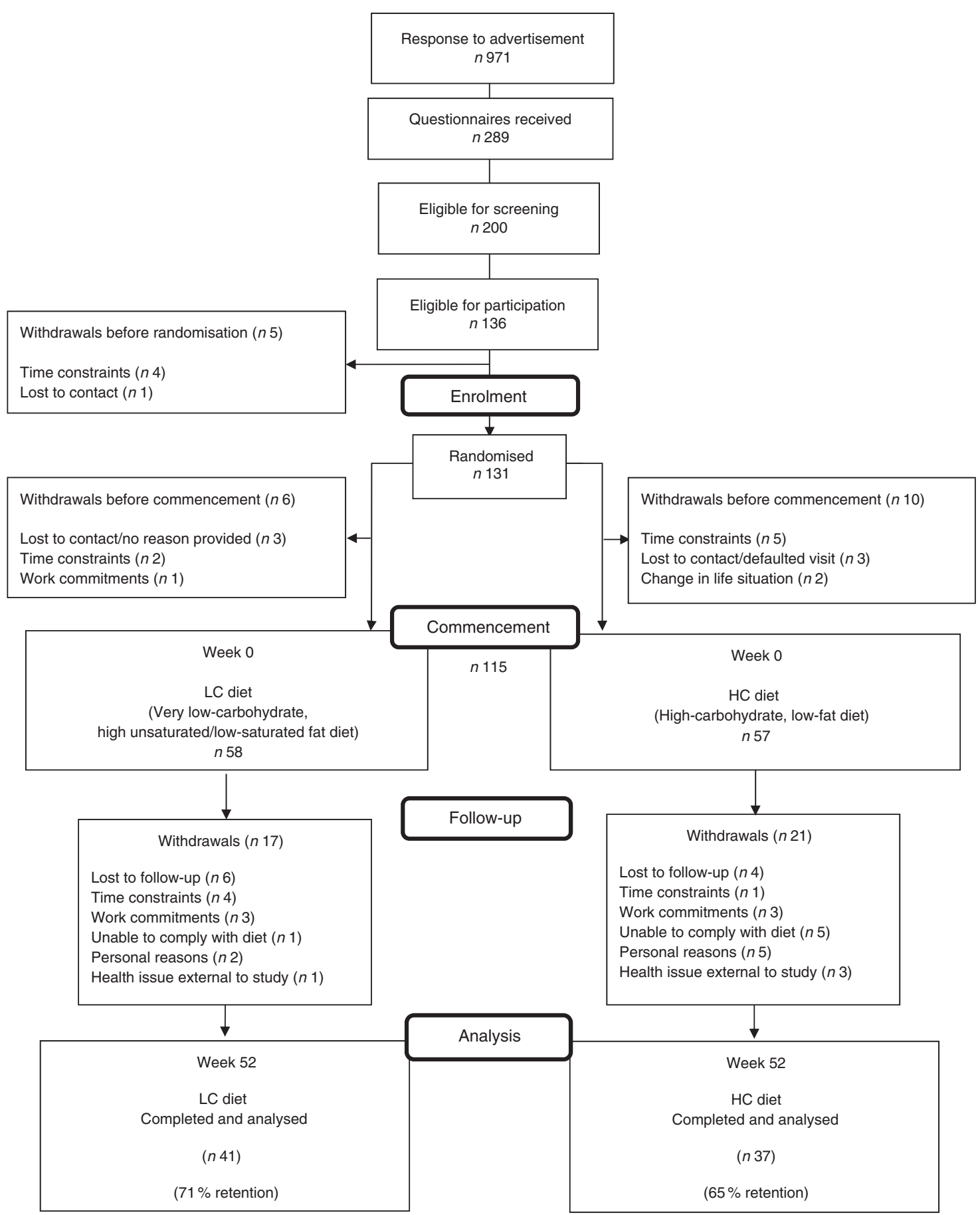

Fig. 1. The study flow diagram.

verbal fluency test performance in adults with T2D, the effect sizes (for time) were comparatively smaller (Cohen's $d=0 \cdot 2)^{(26)}$, mean annual decline of $0 \cdot 4$ points or $0 \cdot 1 \pm 1$ sD units ${ }^{(32)}$, and these effects emerged after 4-6 years. Therefore, given the 12-month time frame of the present study and magnitude of the effect observed, it is possible that the decline was due to increasing test difficulty and poor test equivalence that could be considered a limitation of the test battery design. Future studies should be cautious of these methodological issues.
A strength of the study was the use of a cognitive test battery that was more comprehensive and assessed multiple cognitive domains compared with previous studies examining LC and HC diets, particularly in individuals with T2D. However, the study has several limitations. The absence of a non-intervention, control group makes it difficult to decipher the effects observed overtime between practice effects relating to repeated testing or the direct effect of weight loss and the lifestyle intervention. The study also examined a relatively modest sample size. However, 
Table 3. Estimated marginal means derived using linear mixed-effects model analysis for cognitive performance across 52 weeks for low-carbohydrate (LC) diet and high-carbohydrate (HC) diet groups ${ }^{\star}$ (Mean values and $95 \%$ confidence intervals)

\begin{tabular}{|c|c|c|c|c|c|c|c|c|c|c|c|c|c|c|c|c|c|c|c|}
\hline & \multicolumn{8}{|c|}{ LC diet } & \multicolumn{8}{|c|}{$\mathrm{HC}$ diet } & \multirow{2}{*}{\multicolumn{2}{|c|}{$\begin{array}{l}\text { Difference in mean } \\
\text { changes between } \\
\text { groups }\end{array}$}} & \multirow[b]{3}{*}{$P($ diet $\times$ time $)$} \\
\hline & \multicolumn{2}{|r|}{ Week 0} & \multicolumn{2}{|c|}{ Week 24} & \multicolumn{2}{|c|}{ Week 52} & \multicolumn{2}{|c|}{$\begin{array}{l}\text { Change over } \\
52 \text { weeks }\end{array}$} & \multicolumn{2}{|r|}{ Week 0} & \multicolumn{2}{|r|}{ Week 24} & \multicolumn{2}{|r|}{ Week 52} & \multicolumn{2}{|c|}{$\begin{array}{l}\text { Change over } \\
52 \text { weeks }\end{array}$} & & & \\
\hline & Mean & $95 \% \mathrm{Cl}$ & Mean & $95 \% \mathrm{Cl}$ & Mean & $95 \% \mathrm{Cl}$ & Mean & $95 \% \mathrm{Cl}$ & Mean & $95 \% \mathrm{Cl}$ & Mean & $95 \% \mathrm{Cl}$ & Mean & $95 \% \mathrm{Cl}$ & Mean & $95 \% \mathrm{Cl}$ & Mean & $95 \% \mathrm{Cl}$ & \\
\hline DSST score & $51 \cdot 2$ & $48 \cdot 3,54 \cdot 1$ & 51.1 & $47 \cdot 9,54 \cdot 3$ & 53.5 & $50 \cdot 7,56 \cdot 3$ & $2 \cdot 30$ & $0.39,4.21$ & 53.0 & $50 \cdot 2,55 \cdot 9$ & 53.8 & $50.7,57.0$ & 54.1 & $51 \cdot 3,56.9$ & $1 \cdot 10$ & $-0.81,3.0$ & 1.20 & $-1.5,3.9$ & 0.28 \\
\hline $\begin{array}{l}\text { Letter sets } \\
\text { score }\end{array}$ & 8.55 & $7 \cdot 74,9 \cdot 37$ & 8.79 & $7 \cdot 84,9 \cdot 75$ & 9.10 & $8 \cdot 21,10 \cdot 0$ & 0.55 & $-0.09,1 \cdot 19$ & 9.65 & $8 \cdot 83,10.5$ & 9.96 & $9.02,10.9$ & 9.74 & $8 \cdot 84,10 \cdot 6$ & 0.10 & $-0.55,0.74$ & 0.45 & $-0.45,1.36$ & 0.48 \\
\hline $\begin{array}{l}\text { Operation span } \\
\text { score }\end{array}$ & 29.6 & $27 \cdot 6,31 \cdot 7$ & 29.8 & $27 \cdot 3,32 \cdot 4$ & $31 \cdot 1$ & $28 \cdot 8,33 \cdot 3$ & 1.44 & $-0 \cdot 28,3 \cdot 16$ & 30.7 & $28 \cdot 7,32 \cdot 7$ & 29.4 & $26 \cdot 9,31 \cdot 9$ & 30.3 & $28 \cdot 0,32 \cdot 6$ & $-0 \cdot 45$ & $-2 \cdot 24,1 \cdot 35$ & 1.88 & $-0.60,4.37$ & 0.24 \\
\hline $\begin{array}{l}\text { Word memory } \\
\text { score }\end{array}$ & 6.89 & $6.39,7.40$ & 6.57 & $6 \cdot 00,7 \cdot 14$ & 6.39 & $5 \cdot 82,6.96$ & -0.51 & $-1.05,0.03$ & 7.14 & $6.63,7.65$ & 6.39 & $5.83,6.95$ & 6.51 & $5.93,7.09$ & -0.63 & $-1 \cdot 18,-0.08$ & 0.12 & $-0.65,0.89$ & 0.53 \\
\hline $\begin{array}{l}\text { 2-Choice } \\
\text { reaction time } \\
\text { score }\end{array}$ & 1.41 & $1.33,1.49$ & 1.40 & $1.31,1.48$ & 1.47 & $1.39,1.55$ & 0.06 & $-0.007,0.13$ & 1.44 & $1.36,1.52$ & 1.43 & $1.34,1.51$ & 1.45 & $1.36,1.53$ & 0.004 & $-0.07,0.08$ & 0.06 & $-0.04,0.16$ & 0.41 \\
\hline OMO score & 0.57 & $0.51,0.62$ & 0.59 & $0.54,0.64$ & 0.65 & $0.59,0.71$ & 0.08 & $0.04,0.13$ & 0.60 & $0.54,0.66$ & 0.60 & $0.55,0.65$ & 0.67 & $0.61,0.73$ & 0.07 & $0.02,0.11$ & 0.01 & $-0.05,0.08$ & 0.83 \\
\hline MScan score & 0.97 & $0.91,1.02$ & 0.90 & $0.84,0.96$ & 1.01 & $0.95,1.07$ & 0.04 & $0.004,0.08$ & 0.95 & $0.90,1.01$ & 0.91 & $0.85,0.97$ & 0.99 & $0.93,1.05$ & 0.03 & $-0.005,0.07$ & 0.008 & $-0.05,0.06$ & 0.65 \\
\hline $\begin{array}{l}\text { Colour Stroop } \\
\text { score }\end{array}$ & -0.20 & $-0.23,-0.17$ & -0.19 & $-0.23,-0.14$ & -0.17 & $-0.21,-0.13$ & 0.03 & $-0.01,0.07$ & -0.18 & $-0.21,-0.14$ & -0.17 & $-0.21,-0.13$ & -0.14 & $-0.18,-0.10$ & 0.04 & $-0.004,0.08$ & -0.01 & $-0.07,0.05$ & 0.71 \\
\hline
\end{tabular}

LC diet, very low-carbohydrate, high-unsaturated/low-saturated-fat diet; HC diet, high-carbohydrate, low-fat diet; DSST, digit symbol substitution test; OMO, odd-man-out letter test; MScan, number memory scanning test.

* Outcome measures for cognitive tasks described in Table 1. 
previous short-term studies with smaller sample sizes have been sufficient to demonstrate differences in cognitive performance between HC and LC diets ${ }^{(23,24)}$, and the total number of participants recruited exceeded those determined by a priori power analyses. This study was also conducted in a Caucasian, middle-aged, T2D population with relatively good metabolic control and minimal cognitive deficits based on age-specific normative scores ${ }^{(33)}$. However, clinically relevant cognitive decrements in T2D occur mainly in those with diabetes-related vascular complications and older adults $>65$ years of age ${ }^{(7)}$. Therefore, although our participants represent an age group at a critical window of opportunity for targeted interventions that may slow diabetes complication progression, further studies that examine the effects of these dietary patterns in older individuals with diabetes, pre-existing diabetes complications, poorer cognitive function and/or metabolic control and ethnically diverse populations are required to better understand the wider generalisability of the current findings. In addition, it is possible that the tasks used to assess changes in cognitive performance lacked sufficient sensitivity to detect any differences between the diets examined. However, the tests comprising the large battery of cognitive tasks used in this study were selected based on their known sensitivity to dietary manipulation $^{(18)}$, and assessed different cognitive domains sensitive to $\mathrm{T} 2 \mathrm{D}^{(6)}$. This suggests that the current battery would have been suitable to identify any diet differences. Nevertheless, the test battery did not assess all possible cognitive domains, such as long-term/delayed verbal memory, the impairment of which is well known in diabetes ${ }^{(34)}$. Future studies could further extend the cognitive domains tested. The study also examined the cognitive effects of the LC and HC diets when administered as part of prescriptive energy reduced diet plan that achieved substantial weight loss and metabolic improvement. Whether similar responses between the dietary patterns would be observed over a longer follow-up period and with the dietary patterns administered ad libitum requires further investigation.

In summary, in overweight and obese adults with T2D, both LC and HC weight-loss diets combined with exercise training had similar effects on cognitive performance. This suggests that both dietary patterns can be used as a strategy for diabetes and weight management without concerns of negative impacts on cognitive function. In fact, the cognitive improvements observed over time in both diet groups and the modest relationship between weight loss and improvements in cognitive performance demonstrate the potential benefits of combined diet and lifestyle interventions in T2D. Further studies should be undertaken to evaluate the cognitive effects of these diets in older adults with T2D, pre-existing diabetes complications, poorer cognitive function and glycaemic control, who are at greater risk of cognitive decline.

\section{Acknowledgements}

The authors would like to thank the participants for their invaluable help with the study. The authors also gratefully acknowledge the Clinical Research Team at the CSIRO - Food and Nutrition, Adelaide, South Australia: Julia Weaver, Anne
McGuffin and Vanessa Courage for trial coordination; Lindy Lawson and Theresa Mckinnon for nursing expertise; Pennie Taylor, Janna Lutze, Paul Foster, Gemma Williams, Hannah Gilbert and Fiona Barr for assisting in the design and implementing the dietary intervention; Heidi Long and Laura Edney for administering the cognitive tests; Julie Syrette for data management expertise; Kathryn Bastiaans for assisting with cognitive data processing; Kelly French, Jason Delfos, Kristi Lacey-Powell, Marilyn Woods, John Perrin, Simon Pane, Annette Beckette (South Australian Aquatic Centre \& Leisure Centre, South Australia), Angie Mondello and Josh Gniadek (Boot Camp Plus, South Australia), and Luke Johnston and Annie Hastwell (Fit for Success, South Australia) for implementing the exercise intervention.

Funding was provided by a project grant received from National Health and Medical Research Council of Australia (103415). J. T. was supported by a postgraduate research scholarship from the Agency for Science, Technology and Research, Singapore. The funding sponsors had no role in the design, data collection and analysis, the preparation of the manuscript or decision to publish.

G. B. had full access to all of the data in this study and takes complete responsibility for the integrity of the data and the accuracy of the data analysis. Study concept and design: all authors. Data analysis and interpretation: all authors. Manuscript drafting: J. T., I. T. Z. and G. D. B. Critical revision the manuscript for intellectual content: N. D. L.-M., C. H. T., V. D., M. N., J. D. B. and G. A. W. Approval of final manuscript: all authors. Obtained funding: G. D. B., M. N., J. D. B., N. D. L.-M. and C. H. T. Study supervision: G. D. B., N. D. L.-M., C. H. T., M. N. and J. D. B.

The authors declare that there are no conflicts of interest.

\section{References}

1. Evert AB, Boucher JL, Cypress M, et al. (2013) Nutrition therapy recommendations for the management of adults with diabetes. Diabetes Care 36, 3821-3842.

2. Yancy WS Jr, Westman EC, McDuffie JR, et al. (2010) A randomized trial of a low-carbohydrate diet vs orlistat plus a low-fat diet for weight loss. Arch Intern Med 170, $136-145$.

3. Ajala O, English P \& Pinkney J (2013) Systematic review and meta-analysis of different dietary approaches to the management of type 2 diabetes. Am J Clin Nutr 97, 505-516.

4. Tay J, Luscombe-Marsh ND, Thompson CH, et al. (2015) Comparison of low- and high-carbohydrate diets for type 2 diabetes management: a randomized trial. Am J Clin Nutr 102, 780-790.

5. Chung CC, Pimentel D, Jor'dan AJ, et al. (2015) Inflammationassociated declines in cerebral vasoreactivity and cognition in type 2 diabetes. Neurology 85, 450-458.

6. Cukierman T, Gerstein HC \& Williamson JD (2005) Cognitive decline and dementia in diabetes - systematic overview of prospective observational studies. Diabetologia 48, 2460-2469.

7. Biessels GJ, Deary IJ \& Ryan CM (2008) Cognition and diabetes: a lifespan perspective. Lancet Neurol 7, 184-190.

8. Institute of Medicine (2005) Dietary Reference Intakes: Energy, Carbohydrate, Fiber, Fat, Fatty Acids, Cholesterol, Protein, and Amino Acids (Macronutrients). Washington, DC: National Academy of Sciences Press. 
9. Crowe TC (2005) Safety of low-carbohydrate diets. Obes Rev 6, 235-245.

10. Brinkworth GD, Buckley JD, Noakes M, et al. (2009) Longterm effects of a very low-carbohydrate diet and a low-fat diet on mood and cognitive function. Arch Intern Med 169, 1873-1880.

11. Makris A, Darcey VL, Rosenbaum DL, et al. (2013) Similar effects on cognitive performance during highand low-carbohydrate obesity treatment. Nutr Diabetes 3, e89.

12. Rodbard HW, Blonde L, Braithwaite SS, et al. (2007) American Association of Clinical Endocrinologists medical guidelines for clinical practice for the management of diabetes mellitus. Endocr Pract 13, Suppl. 1, 1-68.

13. Colberg SR, Albright AL, Blissmer BJ, et al. (2010) Exercise and type 2 diabetes: American College of Sports Medicine and the American Diabetes Association: joint position statement. Exercise and type 2 diabetes. Med Sci Sports Exerc 42, 2282-2303

14. Wechsler D (1981) Manual for the Wechsler Adult Intelligence Scale - Revised. New York, NY: The Psychological Corporation.

15. Ekstrom R, French J, Harman H, et al. (1976) Kit of Factor Referenced Cognitive Tests. Princeton, NJ: Educational Testing Service.

16. Danthiir V, Burns NR, Nettelbeck T, et al. (2011) The older people, omega-3, and cognitive health $(\mathrm{EPOCH})$ trial design and methodology: a randomised, double-blind, controlled trial investigating the effect of long-chain omega- 3 fatty acids on cognitive ageing and wellbeing in cognitively healthy older adults. Nutr J 10, 117.

17. Danthiir V, Hosking D, Burns NR, et al. (2014) Cognitive performance in older adults is inversely associated with fish consumption but not erythrocyte membrane $n$ - 3 fatty acids. J Nutr 144, 311-320.

18. Hoyland A, Lawton CL \& Dye L (2008) Acute effects of macronutrient manipulations on cognitive test performance in healthy young adults: a systematic research review. Neurosci Biobehav Rev 32, 72-85.

19. van den Berg E, Kloppenborg RP, Kessels RP, et al. (2009) Type 2 diabetes mellitus, hypertension, dyslipidemia and obesity: a systematic comparison of their impact on cognition. Biochim Biophys Acta 1792, 470-481.

20. Beck AT, Steer RA \& Brown GK (1996) Manual of the Beck Depression Inventory- II. San Antonio, TX: Psychological Corporation.
21. Faul F, Erdfelder E, Buchner A, et al. (2009) Statistical power analyses using G*Power 3.1: tests for correlation and regression analyses. Behav Res Methods 41, 1149-1160.

22. Cohen J (1988) Statistical Power Analysis for the Behavioral Sciences, 2nd ed. New York: Academic Press.

23. D'Anci KE, Watts KL, Kanarek RB, et al. (2009) Lowcarbohydrate weight-loss diets. Effects on cognition and mood. Appetite 52, 96-103.

24. Halyburton AK, Brinkworth GD, Wilson CJ, et al. (2007) Lowand high-carbohydrate weight-loss diets have similar effects on mood but not cognitive performance. Am J Clin Nutr $\mathbf{8 6}$, 580-587.

25. Machulda MM, Pankratz VS, Christianson TJ, et al. (2013) Practice effects and longitudinal cognitive change in normal aging vs. incident mild cognitive impairment and dementia in the Mayo Clinic Study of Aging. Clin Neuropsychol 27, 1247-1264.

26. van den Berg E, Reijmer YD, de Bresser J, et al. (2010) A 4 year follow-up study of cognitive functioning in patients with type 2 diabetes mellitus. Diabetologia 53, 58-65.

27. Lowe C \& Rabbitt P (1998) Test/re-test reliability of the CANTAB and ISPOCD neuropsychological batteries: theoretical and practical issues. Cambridge Neuropsychological Test Automated Battery. International Study of Post-Operative Cognitive Dysfunction. Neuropsychologia 36, 915-923.

28. Chang YK, Pan CY, Chen FT, et al. (2012) Effect of resistanceexercise training on cognitive function in healthy older adults: a review. J Aging Phys Act 20, 497-517.

29. Bherer L, Erickson KI \& Liu-Ambrose T (2013) A review of the effects of physical activity and exercise on cognitive and brain functions in older adults. J Aging Res 2013, 657508.

30. Blondell SJ, Hammersley-Mather R \& Veerman JL (2014) Does physical activity prevent cognitive decline and dementia?: a systematic review and meta-analysis of longitudinal studies. BMC Public Health 14, 510.

31. Witte AV, Fobker M, Gellner R, et al. (2009) Caloric restriction improves memory in elderly humans. Proc Natl Acad Sci US A 106, $1255-1260$.

32. Christman AL, Matsushita K, Gottesman RF, et al. (2011) Glycated haemoglobin and cognitive decline: the Atherosclerosis Risk in Communities (ARIC) study. Diabetologia 54, 1645-1652.

33. Hoyer WJ, Stawski RS, Wasylyshyn C, et al. (2004) Adult age and digit symbol substitution performance: a meta-analysis. Psychol Aging 19, 211-214.

34. Awad N, Gagnon M \& Messier C (2004) The relationship between impaired glucose tolerance, type 2 diabetes, and cognitive function. J Clin Exp Neuropsychol 26, 1044-1080. 